Ján Borsík, Matematický ústav SAV, Grešákova 6, 04001 Košice, Slovakia

Jaroslav Červeňanský, Katedra matematickej analýzy MFF UK, Univerzita

Komenského, Mlynská dolina, 84215 Bratislava, Slovakia

Tibor Šalát, Katedra algebry a teórie čísel MFF UK, Univerzita

Komenského, Mlynská dolina, 84215 Bratislava, Slovakia

\title{
REMARKS ON FUNCTIONS PRESERVING CONVERGENCE OF INFINITE SERIES
}

\begin{abstract}
A function $f: \mathbb{R} \rightarrow \mathbb{R}$ preserves absolute convergence of series if for each absolutely convergent series $\sum_{n=1}^{\infty} a_{n}$ its $f$-transform $\sum_{n=1}^{\infty} f\left(a_{n}\right)$ is absolutely convergent. In this note, we shall study functions that preserve absolute convergence of series.
\end{abstract}

\section{Introduction}

In papers [2], [4], [5] (Also see [3], pages 84-87.) functions $f: \mathbb{R} \rightarrow \mathbb{R}$ preserving convergence of infinite series are studied. The concept of such functions can be introduced in this manner: If $\sum_{n=1}^{\infty} a_{n}$ is a series of real numbers and $f: \mathbb{R} \rightarrow \mathbb{R}$, then the series $\sum_{n=1}^{\infty} f\left(a_{n}\right)$ is called the $f$-transform of the series $\sum_{n=1}^{\infty} a_{n}$. A function $f: \mathbb{R} \rightarrow \mathbb{R}$ is said to be a convergence preserving function provided that $\sum_{n=1}^{\infty} f\left(a_{n}\right)$ is convergent whenever $\sum_{n=1}^{\infty} a_{n}$ converges.

In the paper [1] functions preserving convergence of series from certain classes of series are investigated.

The purpose of these remarks is to extend these investigations. For example we shall study functions that preserve absolute convergence of series. A function $f$ preserves absolute convergence of series if for each absolutely convergent series $\sum_{n=1}^{\infty} a_{n}$ its $f$-transform $\sum_{n=1}^{\infty} f\left(a_{n}\right)$ is absolutely convergent.

Denote by $F^{(c p)}$ and $F^{(a c p)}$ the class of all $f: \mathbb{R} \rightarrow \mathbb{R}$ that preserve convergence and absolute convergence of series, respectively.

Key Words: Function preserving convergence of infinite series, Baire category

Mathematical Reviews subject classification: Primary: 26A99

Received by the editors March 22, 1995 
In connection with classes $F^{(c p)}$ and $F^{(a c p)}$ the idea of investigating similar classes $F^{(c, a c)}, F^{(a c, c)}$ arises. Here $F^{(c, a c)}, F^{(a c, c)}$ denotes the class of all $f: \mathbb{R} \rightarrow \mathbb{R}$ such that $\sum_{n=1}^{\infty}\left|f\left(a_{n}\right)\right|<\infty$ whenever $\sum_{n=1}^{\infty} a_{n}$ converges and $\sum_{n=1}^{\infty} f\left(a_{n}\right)$ converges whenever $\sum_{n=1}^{\infty}\left|a_{n}\right|<\infty$, respectively. In the sense of this notation the class $F^{(c p)}$ could be denoted by $F^{(c, c)}$ and $F^{(a c p)}$ by $F^{(a c, a c)}$.

\section{Fundamental properties of classes $F^{(c p)}, F^{(a c p)}, F^{(c, a c)}$, $F^{(a c, c)}$}

The following fundamental result proved originally in [2] by R. Rado (Also see [3], pages 84-87, [4], [5].) describes the form of functions of the class $F^{(c p)}$.

Theorem A A function $f: \mathbb{R} \rightarrow \mathbb{R}$ belongs to $F^{(c p)}$ if and only if there are $a \in \mathbb{R}$ and $\delta>0$ such that for each $x \in(-\delta, \delta)$ we have $f(x)=a x$.

Theorem A will be often used in the following form. From Theorem A we get the following proposition.

Proposition 2.1 We have $F^{(c p)} \subset F^{(a c p)}$.

Proof. If $f \in F^{(c p)}$, then according to Theorem A we have $f(x)=a x$ for $x \in$ $(-\delta, \delta)$. It is now easy to check that if $\sum_{n=1}^{\infty}\left|a_{n}\right|<\infty$, then $\sum_{n=1}^{\infty}\left|f\left(a_{n}\right)\right|<\infty$.

Remark 2.2 The inclusion in Proposition 2.1 is proper. This fact will be shown in the following examples.

Example 2.3 Put $f(0)=0, f(x)=x \sin \frac{1}{x}$ for $x \neq 0$. Then evidently $|f(x)| \leq|x|$ for each $x \in \mathbb{R}$ and so $f \in F^{(a c p)}$. But in view of Theorem A the function $f$ does not belong to $F^{(c p)}$.

Example 2.4 Let $a$ be a non-zero real number. Define $g: \mathbb{R} \rightarrow \mathbb{R}$ by

$$
g(x)= \begin{cases}0 & \text { for } x \text { irrational } \\ a x & \text { for } x \text { rational. }\end{cases}
$$

Then $g \in F^{(a c p)} \backslash F^{(c p)}$.

Example 2.5 It is easy to verify that the function $f(x)=a|x|^{\alpha}(a, \alpha$ are positive constants) belongs to $F^{(a c p)}$ if and only if $\alpha \geq 1$. 
The foregoing examples show that for $F^{(a c p)}$ a result analogous to Theorem A does not hold. Even by a little modification of Example 2.3 we get a function belonging to $F^{(a c p)}$ the four Dini derivatives of which at the point 0 are distinct. Of course all these Dini derivatives are finite as can be seen from the following result.

Theorem 2.6 Let $f: \mathbb{R} \rightarrow \mathbb{R}$ be a function. Then the following conditions are equivalent:

(i) $f \in F^{(a c p)}$,

(ii) $f \in F^{(a c, c)}$,

(iii) $f(0)=0$ and $\limsup _{x \rightarrow 0}|f(x) / x|<\infty$.

Proof. (i) $\Rightarrow$ (ii) is obvious.

(ii) $\Rightarrow$ (iii). Since the series $0+0+\cdots+0+\ldots$ converges absolutely, the series $\sum_{n=1}^{\infty} f(0)$ converges and so $f(0)=0$. We prove the second part of (iii). Suppose that

$$
\liminf _{x \rightarrow 0-} f(x) / x=-\infty
$$

Then for each $n \in \mathbb{N}$ there exists an $x_{n} \in\left(-1 / n^{2}, 0\right)$ such that

$$
\frac{f\left(x_{n}\right)}{x_{n}}<-n
$$

Note that from this we have $f\left(x_{n}\right)>0$ and $-1 / n^{2} x_{n}>1$. Denote by $v_{n}$ the least positive integer such that

$$
v_{n} \geq-\frac{1}{n^{2} x_{n}}
$$

The definition of $v_{n}$ yields $v_{n}-1<-1 / n^{2} x_{n}$ and hence

$$
-v_{n} x_{n}<-x_{n}+\frac{1}{n^{2}}<\frac{2}{n^{2}} \text {. }
$$

Construct the series

$$
\underbrace{x_{1}+\cdots+x_{1}}_{v_{1} \text {-times }}+\underbrace{x_{2}+\cdots+x_{2}}_{v_{2} \text {-times }}+\cdots+\underbrace{x_{n}+\cdots+x_{n}}_{v_{n} \text {-times }}+\cdots=\sum_{k=1}^{\infty} a_{k} .
$$


This series converges absolutely because of (3) but the series $\sum_{k=1}^{\infty} f\left(a_{k}\right)$ diverges since, according to (1) and (2), we have

$$
\sum_{k=1}^{\infty} f\left(a_{k}\right)=\sum_{n=1}^{\infty} v_{n} f\left(x_{n}\right) \geq \sum_{n=1}^{\infty}\left(-\frac{1}{n^{2} x_{n}}\right)\left(-n x_{n}\right)=\sum_{n=1}^{\infty} \frac{1}{n}=\infty .
$$

Therefore $\liminf _{x \rightarrow 0-} f(x) / x>-\infty$. Similarly it can be proved that $\liminf _{x \rightarrow 0+} f(x) / x$ $>-\infty, \limsup f(x) / x<\infty$ and $\limsup f(x) / x<\infty$. Hence we have (iii). $x \rightarrow 0-$

(iii) $\Rightarrow$ (i). There are a $K>0$ and $\delta>0$ such that $f(x) \leq K|x|$ for $|x|<\delta$. Let $\sum_{n=1}^{\infty}\left|a_{n}\right|<\infty$. Then there is an $m$ such that $\left|a_{n}\right|<\delta$ for $n \geq m$. Then $\sum_{n=m}^{\infty}\left|f\left(a_{n}\right)\right| \leq K \sum_{n=m}^{\infty}\left|a_{n}\right|<\infty$ and $\sum_{n=1}^{\infty}\left|f\left(a_{n}\right)\right|<\infty$, as well. Hence $f$ belongs to $F^{(a c p)}$.

Remark 2.7 We see from Theorem 2.6 that each $f \in F^{(a c p)}$ is continuous at the point 0. Example 2.4 shows that the set of continuity points of such function can be singleton.

In [5] it is noted that $F^{(c p)}$ is a linear space (with operation + and multiplication by real numbers in the usual way). For the class $F^{(a c p)}$ we can prove a stronger result.

Theorem 2.8 The class $F^{(a c p)}$ is an algebra (of functions) closed with respect to the composition of functions.

Proof. Obviously $F^{(a c p)}$ is a linear space. Further, if $f, g \in F^{(a c p)}$, then $g \cdot f$ and also $g \circ f$ belong to $F^{(a c p)}$. This is easy to shown. Let $\sum_{n=1}^{\infty}\left|a_{n}\right|<$ $\infty$. Then by assumption $\sum_{n=1}^{\infty}\left|f\left(a_{n}\right)\right|<\infty$. Since $\sum_{n=1}^{\infty}\left|g\left(a_{n}\right)\right|<\infty$, the sequence $\left\{\left|g\left(a_{n}\right)\right|\right\}_{n=1}^{\infty}$ is bounded, say $\left|g\left(a_{n}\right)\right| \leq K$ for each $n \in \mathbb{N}$. Hence $\sum_{n=1}^{\infty}\left|(g \cdot f)\left(a_{n}\right)\right|=\sum_{n=1}^{\infty}\left|g\left(a_{n}\right)\right| \cdot\left|f\left(a_{n}\right)\right| \leq K \sum_{n=1}^{\infty}\left|f\left(a_{n}\right)\right|<\infty$. Similarly $\sum_{n=1}^{\infty}\left|(g \circ f)\left(a_{n}\right)\right|=\sum_{n=1}^{\infty}\left|g\left(f\left(a_{n}\right)\right)\right|<\infty$.

Remark 2.9 It can be easily verified that also the class $F^{(c p)}$ is closed with respect to composition of functions.

Note that $F^{(c, a c)} \subset F^{(c p)}$. We give a characterization of the class $F^{(c, a c)}$.

Theorem 2.10 A function $f: \mathbb{R} \rightarrow \mathbb{R}$ belongs to $F^{(c, a c)}$ if and only if it is identically equal to zero in a neighborhood of 0 .

Proof. If $f(x)=0$ in a neighborhood of 0 , then obviously $f$ belongs to $F^{(c, a c)}$. Conversely, let $f \in F^{(c, a c)}$. Then the function $f$ belongs to $F^{(c p)}$ and so by Theorem A we have $f(x)=a x$ in a neighborhood $(-\delta, \delta)(\delta>0)$ of 0 . 
If $a \neq 0$, choose an $m$ such that $1 / m<\delta$. Then the series $\sum_{n=m}^{\infty}(-1)^{n-1} / n$ converges but its $f$-transform $a \sum_{n=m}^{\infty}(-1)^{n-1} / n$ is not absolutely convergent. Hence we have $a=0$ and the theorem follows.

Remark 2.11 From previous results we get the following relations

$$
F^{(c, a c)} \subset F^{(c p)} \subset F^{(a c p)}=F^{(a c, c)}
$$

The first of these inclusions is proper by Theorem A and Theorem 2.6 the second inclusion is proper also by Theorem A and Theorem 2.6 (see also Examples $2.3-2.5)$.

Finally, let us remark that $F^{(a c p)}$ is an algebra (Theorem 2.8), further $F^{(c p)}$ is not an algebra (e.g. $f(x)=x$ belongs to $F^{(c p)}$ but $f^{2}(x)=x^{2}$ does not belong to $F^{(c p)}$ - see Theorem A). It can be easily checked that $F^{(c, a c)}$ is an algebra (Theorem 2.10) and that $F^{(c, a c)}$ is closed with respect to composition of functions. Hence all classes in (4) are closed under the composition of functions.

\section{Topological properties of classes $F^{(c, a c)}, F^{(c p)}$ and $F^{(a c p)}$}

We shall consider $H=\left\{f: \mathbb{R} \rightarrow \mathbb{R}: \lim _{x \rightarrow 0} f(x)=0=f(0)\right\}$ as a metric space with the metric $\rho$ of uniform convergence, $\rho\left(f_{1}, f_{2}\right)=\min \left\{1, \sup \left\{\mid f_{1}(x)-\right.\right.$ $\left.\left.f_{2}(x) \mid: x \in \mathbb{R}\right\}\right\}$. This space is complete. ( $H$ is a closed set in the space of all functions $f: \mathbb{R} \rightarrow \mathbb{R}$ with the metric $\rho$.)

In this part of the paper we shall study the position of $F^{(a c p)}$ in $H$ and considering each of the classes $F^{(c p)}, F^{(a c p)}$ as a subspace of $H$ we shall also describe the position of $F^{(c, a c)}$ in $F^{(c p)}$ and $F^{(c p)}$ in $F^{(a c p)}$. In first place we shall describe the position of the set $F^{(a c p)}$ in the space $H$.

Theorem 3.1 The set $F^{(a c p)}$ is a $F_{\sigma}$-set of the first Baire category in the space $H$.

Proof. For $k, m \in \mathbb{N}$ we put

$$
F_{k, m}=\{f \in H: \forall x \in(-1 / m, 1 / m):|f(x)| \leq k|x|\} .
$$

It is easy to check that for fixed $k, m$ the set $F_{k, m}$ is closed in $H$. Further, according to Theorem 2.6 we have

$$
F^{(a c p)}=\bigcup_{k=1}^{\infty} \bigcup_{m=1}^{\infty} F_{k, m} .
$$


Hence $F^{(a c p)}$ is an $\mathrm{F}_{\sigma}$-set in $H$.

We prove that for fixed $k, m$ the set $F_{k, m}$ is nowhere dense in $H$. Since $F_{k, m}$ is closed, it suffices to prove that it is a co-dense set. To prove this it is suffices to show that each ball $K(f, \varepsilon)=\{h \in H: \rho(f, h)<\varepsilon\}(\varepsilon>0)$ of $H$ contains an element of $H \backslash F_{k, m}$. We can assume that $f \in F_{k, m}$. Put $\delta=\min \{1 / m, \varepsilon / 4 k\}$ and define $g: \mathbb{R} \rightarrow \mathbb{R}$ by

$$
g(x)= \begin{cases}2 k x & \text { for } x \in(-\delta, \delta) \\ f(x) & \text { otherwise }\end{cases}
$$

Then for $|x|<\delta$ we have $|f(x)-g(x)| \leq|f(x)|+|g(x)| \leq k|x|+2 k|x|<3 k \delta$ and hence $\rho(f, g) \leq 3 k \delta<\varepsilon$. Thus $g$ belongs to $K(f, \varepsilon)$ and simultaneously it belongs to $H \backslash F_{k, m}$ by definition of $F_{k, m}$. From (5) we now see that $F^{(a c p)}$ is a set of the first category in $H$.

Remark 3.2 By a small modification of the proof of Theorem 3.1 we can show that the set $F^{(a c p)}$ is of the first category even in the space $\left(F^{(a c p)}, \rho\right)$.

The following theorem ends our topological investigation concerning the classes $F^{(c, a c)}, F^{(c p)}$ and $F^{(a c p)}$.

Theorem 3.3 (i) The set $F^{(c, a c)}$ is dense and co-dense in each of the metric spaces $F^{(c p)}, F^{(a c p)}$ and $H$.

(ii) The set $F^{(c p)}$ is dense and co-dense in each of the metric spaces $F^{(a c p)}$ and $H$.

(iii) The set $F^{(a c p)}$ is dense and co-dense in $H$.

Proof. It is easy to see that it is suffices to prove the following statements:

a) the set $F^{(c, a c)}$ is dense in $H$,

b) the set $F^{(c, a c)}$ is co-dense in $F^{(c p)}$,

c) the set $F^{(c p)}$ is co-dense in $F^{(a c p)}$,

d) the set $F^{(a c p)}$ is co-dense in $H$.

We prove a)-d).

a) Let $f \in H$ and $\varepsilon>0$. Then $f(0)=0$ and $f$ is continuous at 0 . Therefore there exists a $\delta>0$ such that $|f(x)|<\varepsilon / 2$ for $|x|<\delta$. Put

$$
g(x)= \begin{cases}0 & \text { if }|x|<\delta \\ f(x) & \text { otherwise }\end{cases}
$$


Then $g \in F^{(c, a c)}$ by Theorem 2.10 and $\rho(f, g)<\varepsilon$.

b) Let $f \in F^{(c, a c)}$ and $\varepsilon>0$. Then according to Theorem 2.10 there is a $\delta>0$ such that $f(x)=0$ for $|x|<\delta$. Put $g(x)= \begin{cases}x & \text { if }|x|<\min \{\delta, \varepsilon / 2\} \\ f(x) & \text { otherwise. }\end{cases}$ Then $g \in F^{(c p)} \backslash F^{(c, a c)}$ by Theorem A and Theorem 2.10 and simultaneously $\rho(f, g)<\varepsilon$.

c) Let $f \in F^{(c p)}$ and $\varepsilon>0$. Then according to Theorem A there is a $\delta>0$ and $k \in \mathbb{R}$ such that $f(x)=k x$ if $|x|<\delta$. Put $\eta=\min \{\delta, \varepsilon / 2 k\}$. Define

$$
g(x)= \begin{cases}k x & \text { for } x \in(-\eta, \eta) \cap \mathbb{Q} \\ 0 & \text { for } x \in(-\eta, \eta) \backslash \mathbb{Q} \\ f(x) & \text { otherwise }\end{cases}
$$

where $\mathbb{Q}$ is the set of all rationals numbers. By Theorem A and Theorem 2.6 the function $g$ belongs to $F^{(a c p)} \backslash F^{(c p)}$ and obviously $\rho(f, g)<\varepsilon$.

d) Since $H$ is a complete metric space and $F^{(a c p)}$ is a set of the first category in $H$, by Theorem 3.1 , the set $F^{(a c p)}$ is co-dense in $H$.

\section{References}

[1] T. Neubrunn and T. Salát, On certain spaces of transformations of infinite series, Časopis Pěst. Mat., 92 (1967), 267-282.

[2] R. Rado, A theorem on infinite series, J. London Math. Soc., XXXV (1960), 273-276.

[3] T. Šalát, Infinite Series (in Slovak), Academia, Prague, 1974.

[4] A. Smith, Convergence preserving functions: an alternate discussion, Amer. Math. Monthly, 98 (1991), 831-833.

[5] G. Wildenberg, Convergence preserving functions, Amer. Math. Monthly, 95 (1988), 542-544. 